\title{
Simple Trapezoidal Recursive Convolution Technique for the Frequency-Dependent FDTD Analysis of a Drude-Lorentz Model
}

\author{
Jun Shibayama, Member, IEEE, Ryoji Ando, Akifumi Nomura, Junji Yamauchi, Senior Member, IEEE, and
} Hisamatsu Nakano, Fellow, IEEE

\begin{abstract}
A concise formulation of the frequency-dependent finite-difference time-domain (FDTD) method is presented using the trapezoidal recursive convolution (TRC) technique for the analysis of a Drude-Lorentz model. The TRC technique requires single convolution integral in the formulation as in the recursive convolution (RC) technique, while maintaining the accuracy comparable to the piecewise linear RC (PLRC) technique with two convolution integrals. The TRC technique is introduced not only to the traditional explicit FDTD, but also to the unconditionally stable implicit FDTD based on the locally one-dimensional (LOD) scheme. Through the analysis of a surface plasmon waveguide, the effectiveness of the TRC technique is investigated for both explicit FDTD and LOD-FDTD, along with the existing RC and PLRC techniques.
\end{abstract}

Index Terms-Finite-difference time-domain (FDTD), piecewise linear recursive convolution (PLRC), surface plasmon polariton, trapezoidal recursive convolution (TRC).

\section{INTRODUCTION}

$\mathbf{R}$ ECENTLY, considerable effort has been directed at analyzing plasmonic devices composed of a metal and a dielectric material [1]. The metal dispersion has frequently been taken into account using the Drude model. To treat the Drude model in the time-domain analysis, we often utilize the frequency-dependent finite-difference time-domain (FDTD) method based on the recursive convolution (RC) and piecewise linear RC (PLRC) techniques [2], [3]. Although the PLRC technique attains higher accuracy than the $\mathrm{RC}$ technique, it requires complicated formulations of two convolution integrals. On the other hand, the trapezoidal RC (TRC) technique provides the accuracy comparable to the PLRC counterpart, while requiring only a single convolution integral as in the RC technique [4], [5]. Note, however, that the application of the TRC technique has been limited to the Debye and Lorentz models, each of which cannot fully express the dispersion of the metal in optical wavelengths, and no attempt has been made to apply the TRC technique to the Drude model frequently used for plasmonic devices.

In this letter, we introduce the TRC technique into the Drude-Lorentz model with multiple Lorentzian poles. The addition of the Lorentz term can accurately account for the

Manuscript received September 02, 2008; revised October 11, 2008. First published November 21, 2008; current version published January 14, 2009. This work was supported in part by MEXT, Grant-in-Aid for Scientific Research (c) (19560355).

The authors are with the Faculty of Engineering, Hosei University, Tokyo 184-8584, Japan (e-mail: shiba@ hosei.ac.jp).

Digital Object Identifier 10.1109/LPT.2008.2009003 measured permittivity of Au and Ag over a wide spectrum from visible to near infrared regions [6]-[12]. While electric field values from two time steps are required due to the presence of the Drude model, the use of the TRC technique similar to the RC technique greatly facilitates the frequency-dependent formulation, offering the accuracy comparable to the PLRC counterpart. We discuss the TRC formulation along with the existing RC and PLRC techniques. We further introduce the TRC technique to the unconditionally stable implicit FDTD based on the locally one-dimensional (LOD) scheme [12], [13]. The effectiveness of the TRC technique is investigated through the analysis of the surface plasmon waveguide.

\section{FORMULATION}

The linear polarization $\boldsymbol{P}(\omega)=\chi(\omega) \boldsymbol{E}(\omega)$, where $\chi(\omega)$ is susceptibility, is represented in the time domain as

$$
\boldsymbol{P}(n \Delta t)=\int_{0}^{n \Delta t} \chi(\tau) \boldsymbol{E}(n \Delta t-\tau) d \tau .
$$

For the RC technique [2], (1) is approximated as

$$
\boldsymbol{P}(n \Delta t)=\sum_{m=0}^{n-1} \boldsymbol{E}^{n-m} \chi^{m}
$$

where $\chi^{m}=\int_{m \Delta t}^{(m+1) \Delta t} \chi(\tau) d \tau$, in which the electric field is assumed constant over $\Delta t$. For the PLRC technique [3], (1) is approximated as

$$
\boldsymbol{P}(n \Delta t)=\sum_{m=0}^{n-1}\left\{\boldsymbol{E}^{n-m} \chi^{m}+\left(\boldsymbol{E}^{n-m-1}-\boldsymbol{E}^{n-m}\right) \xi^{m}\right\}
$$

where $\xi^{m}=(1 / \Delta t) \int_{m \Delta t}^{(m+1) \Delta t}(\tau-m \Delta t) \chi(\tau) d \tau$, in which the electric field has piecewise linear functional dependence over $\Delta t$. Note that the term regarding $\xi^{m}$ appears compared with (2), requiring an additional calculation of the convolution integral. This formulation results in storage of electric field values from two time steps [3]-[5].

For the TRC technique [4], [5], (1) is approximated using an average of the electric fields over two consecutive time steps in the following form:

$$
\boldsymbol{P}(n \Delta t)=\sum_{m=0}^{n-1} \frac{\boldsymbol{E}^{n-m}+\boldsymbol{E}^{n-m-1}}{2} \chi^{m} .
$$

Obviously, the TRC technique requires only a single convolution integral regarding $\chi^{m}$, as in the $\mathrm{RC}$ technique. Note that the efficient TRC technique with electric field values in single time step (single time step TRC) has been developed providing the 
accuracy comparable to the PLRC counterpart, to which only the Debye and Lorentz models are applicable [4], [5]. Unfortunately, the single time step TRC technique is not available for the Drude model, since its susceptibility is not expressed as an exponential function. Nevertheless, as shown below, the TRC technique greatly facilitates the frequency-dependent formulation for the Drude-Lorentz model due to the single convolution integral.

Although any dispersion model is applicable to the analysis with monochromatic sources, here we treat the following Drude-Lorentz model (with $P$ poles for the Lorentz function [2]) to obtain a spectral response in one time solution with the help of a pulse excitation scheme

$$
\varepsilon_{r}(\omega)=\varepsilon_{\infty}+\frac{\omega_{D}^{2}}{j \omega\left(\nu_{D}+j \omega\right)}+\sum_{p=1}^{P} \frac{\Delta \varepsilon G_{p} \omega_{p}^{2}}{j \omega \nu_{p}+\omega_{p}^{2}-\omega^{2}}
$$

where $\varepsilon_{\infty}$ is the dielectric constant of the material at infinite frequency, $\omega$ is the angular frequency, $\omega_{D}$ and $\omega_{p}$ are the electron plasma frequencies, $\nu_{D}$ and $\nu_{p}$ are the effective electron collision frequencies, $G_{p}$ is the pole strength, and $\Delta \varepsilon$ is the weighting coefficient.

Following the RC procedure [2], we newly derive the finitedifference equation to be solved using (4) as

$$
\begin{aligned}
\boldsymbol{E}^{n+1}=\frac{\varepsilon_{\infty}-\frac{\chi^{0}}{2}}{\varepsilon_{\infty}+\frac{\chi^{0}}{2}} \boldsymbol{E}^{n}+\frac{1}{\varepsilon_{\infty}+\frac{\chi^{0}}{2}} \boldsymbol{\phi}^{n} \\
+\frac{\Delta t}{\varepsilon_{0}\left(\varepsilon_{\infty}+\frac{\chi^{0}}{2}\right)}\left(\nabla \times \boldsymbol{H}^{n+1 / 2}\right)
\end{aligned}
$$

where $\chi^{0}=\chi_{D}^{0}+\sum_{p=1}^{P} \operatorname{Re}\left[\chi_{p}^{0}\right]$ and $\boldsymbol{\phi}^{n}=\boldsymbol{\phi}_{D}^{n}+$ $\sum_{p=1}^{P} \operatorname{Re}\left[\phi_{p}^{n}\right] . \boldsymbol{\phi}_{D}^{n}$ and $\boldsymbol{\phi}_{p}^{n}$ are expressed as follows:

$$
\begin{aligned}
\boldsymbol{\phi}_{D}^{n} & =\frac{\boldsymbol{E}^{n}+\boldsymbol{E}^{n-1}}{2} \Delta \chi_{D}^{0}+e^{-\nu_{D} \Delta t} \boldsymbol{\phi}_{D}^{n-1} \\
\boldsymbol{\phi}_{p}^{n} & =\frac{\boldsymbol{E}^{n}+\boldsymbol{E}^{n-1}}{2} \Delta \chi_{p}^{0}+e^{\gamma \Delta t} \boldsymbol{\phi}_{p}^{n-1}
\end{aligned}
$$

where $\gamma=-\alpha+j \beta, \alpha=\nu_{p} / 2$, and $\beta=\sqrt{\omega_{p}^{2}-\alpha^{2}}$. The parameters used for the above equations are given as

$$
\begin{aligned}
\chi_{D}^{0} & =\frac{\omega_{D}^{2}}{\nu_{D}}\left\{\Delta t-\frac{1}{\nu_{D}}\left(1-e^{-\nu_{D} \Delta t}\right)\right\} \\
\chi_{p}^{0} & =-\frac{2 \Delta \varepsilon G_{p} \omega_{p}^{2}}{\gamma\left(\gamma-\gamma^{*}\right)}\left(1-e^{\gamma \Delta t}\right) \\
\Delta \chi_{D}^{0} & =-\frac{\omega_{D}^{2}}{\nu_{D}^{2}}\left(1-e^{-\nu_{D} \Delta t}\right)^{2} \\
\Delta \chi_{p}^{0} & =\chi_{p}^{0}\left(1-e^{\gamma \Delta t}\right)
\end{aligned}
$$

where * represents the complex conjugate. While electric field values from two time steps are required as in the PLRC technique [3], [12], the formulation is quite simple, similar to the RC counterpart [2]. Equation (6) can explicitly be solved combined with the standard finite-difference equations for the $\boldsymbol{H}$ field. Ignoring the terms for the Lorentz model leads to the equations for the Drude model that is applicable only to the near infrared region.

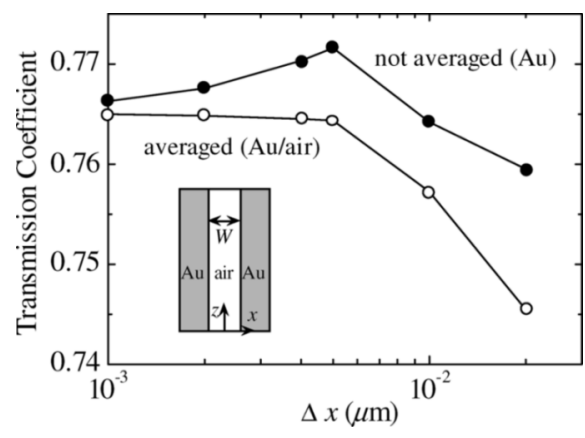

Fig. 1. Transmission coefficient versus $\Delta x$.

We also develop the two-dimensional frequency-dependent LOD-FDTD based on the TRC technique. The equation for TM waves is derived as

$$
\begin{aligned}
E_{z}^{n+1}= & \frac{\varepsilon_{\infty}-\frac{\chi^{0}}{2}}{\varepsilon_{\infty}+\frac{\chi^{0}}{2}} E_{z}^{n}+\frac{1}{\varepsilon_{\infty}+\frac{\chi^{0}}{2}} \phi_{z}^{n} \\
& +\frac{\Delta t}{2 \varepsilon_{0}\left(\varepsilon_{\infty}+\frac{\chi^{0}}{2}\right)}\left(\frac{\partial H_{y}^{\prime}}{\partial x}+\frac{\partial H_{y}^{n+1 / 2}}{\partial x}\right)
\end{aligned}
$$

for the first half step, where the relation $E_{z}^{n+1}=E_{z}^{\prime}$ obtained from the LOD procedure [13] is adopted $\left(H^{\prime}\right.$ and $E^{\prime}$ represent the intermediate fields). For the $H$ field, the standard LODFDTD equation is utilized. The equations for the second half step can be derived similarly. The simplified TRC-LOD equations are solvable as in the PLRC-LOD-FDTD [12].

\section{DISCUSSION}

To investigate the validity of the TRC technique for the Drude-Lorentz model, we analyze the pulse propagation of the TM wave in the air core region sandwiched between the metal claddings, shown in the inset of Fig. 1 [12]. The width of the air core is fixed to be $W=0.2 \mu \mathrm{m}$ and the metal is chosen to be $\mathrm{Au}$ whose parameters are $\varepsilon_{\infty}=5.9673, \omega_{D} / 2 \pi=2113.6 \mathrm{THz}, \omega_{1} / 2 \pi=650.07 \mathrm{THz}$, $\nu_{D} / 2 \pi=15.92 \mathrm{THz}, \nu_{1} / 2 \pi=104.86 \mathrm{THz}$, and $\Delta \varepsilon=1.09$ [6], [9], [12], which corresponds to $P=1$, so that $G_{1}=1$ [2]. We calculate the transmission coefficient obtained from the ratio between the discrete Fourier transforms of the incident pulse and the transmitted pulse after a propagation on length $z=5 \mu \mathrm{m}$. Due to the problem symmetry to the transverse section, only half the section $(x>0)$ is analyzed. No specific absorbing boundary condition is used, since the pulse is tightly confined into the air core region.

Before studying the accuracy of each technique, we emphasize here the necessity of using the averaged permittivity along the interface between a metal and a dielectric material [14]. Throughout this letter, we adopt the averaged permittivity between $\mathrm{Au}$ and air along the interface on which the electric field component is placed. Fig. 1 shows the transmission coefficient at the center wavelength of the pulse $(0.7 \mu \mathrm{m})$ versus transverse sampling width $\Delta x$. For reference, also included is the result with the Au permittivity being applied to the interface (not averaged). The explicit FDTD based on the TRC technique is used, where the upper limit of the Courant-Friedrich-Levy condition $\left(\Delta t_{\mathrm{CFL}}\right)$ is utilized with the longitudinal sampling width being 


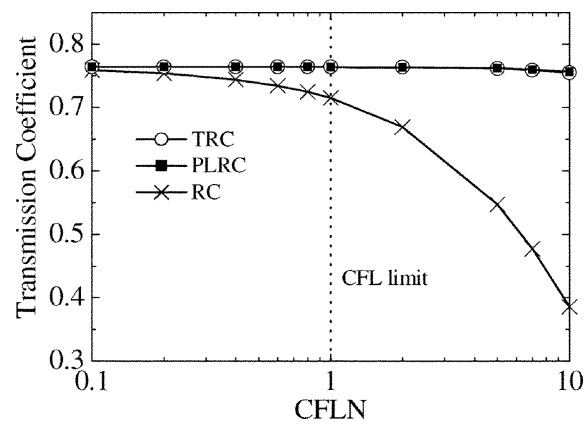

Fig. 2. Transmission coefficient versus CFLN.

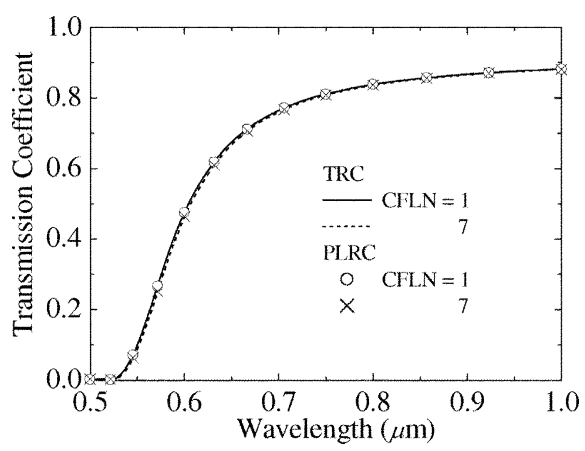

Fig. 3. Transmission coefficient versus wavelength.

fixed to be $\Delta z=0.004 \mu \mathrm{m}$. It is seen that the coefficient obtained with the averaged permittivity monotonically converges, where it becomes almost constant for $\Delta x \leq 0.005 \mu \mathrm{m}$. In contrast, the coefficient without the averaged permittivity does not monotonically converge, primarily because the structure is not accurately modeled [14]. As a result, we have to use the averaged permittivity along the metal/dielectric interface for the solutions of (6) and (7).

We now investigate the accuracy of the TRC technique with respect to the time step size. Here, we use $\Delta x=0.005 \mu \mathrm{m}$ and $\Delta z=0.004 \mu \mathrm{m}$, yielding $\Delta t_{\mathrm{CFL}}=0.0102 \mathrm{fs}\left(\Delta t_{\mathrm{CFL}}=\right.$ $0.00306 \mathrm{fs}$ stated in [12] should be corrected to $0.0102 \mathrm{fs}$ ). The time step size is defined as CFLN $=\Delta t / \Delta t_{\mathrm{CFL}}$. Fig. 2 shows the transmission coefficient versus CFLN at the center wavelength $(0.7 \mu \mathrm{m})$. The results of the RC and PLRC techniques are included for reference. For CFLN $\leq 1$, the explicit FDTD is used, while for CFLN $\geq 1$, the LOD-FDTD is used (the CFL limit is indicated by the dotted line in Fig. 2). It is worth mentioning that the TRC results almost perfectly follow the PLRC results for both explicit and LOD-FDTDs (the RC result degrades with CFLN). For CFLN $=7$, the deviation from the eigenmode solution (0.76451) is only $0.7 \%$. In this case, the computational time of the TRC-LOD-FDTD is reduced to about $30 \%$ of the times of the explicit TRC- and PLRC-FDTDs (the TRC is slightly (a few percent) faster than the PLRC), where a PC with a Pentium 4 processor $(3.46 \mathrm{GHz})$ is used (the degree to which the computational time reduction is attained depends on a computer to be used).

The wavelength response of the transmission coefficient from the LOD-FDTD is depicted in Fig. 3, in which the results of the TRC technique are compared with those of the PLRC technique. Although not shown, the result of the explicit FDTD for
CFLN $=1$ is superimposed on that of the LOD-FDTD for CFLN $=1$. Again, we can find excellent agreement between the TRC and PLRC results, in which the computational time and the required memory are the same level for both techniques. Even for CFLN $=7$, the accuracy is found to be successfully maintained over a wide wavelength range.

\section{CONCLUSION}

We have discussed the effectiveness of the TRC technique for the frequency-dependent FDTD. The formulation for the Drude-Lorentz model is found to be simpler than that of the PLRC technique. We apply the TRC technique not only to the explicit FDTD but also to the unconditionally stable LOD-FDTD. The analysis of the surface plasmon waveguide reveals that the TRC technique provides the accuracy comparable to the PLRC counterpart. Consequently, the FDTD based on the TRC technique could be a practical alternative for an accurate frequency-dependent method of analyzing plasmonic devices. Comparison with the auxiliary differential equation technique for the Drude-Lorentz model [7]-[11] is now under consideration, which will be discussed elsewhere.

\section{REFERENCES}

[1] J. Takahara and F. Kusunoki, "Guiding and nanofocusing of two-dimensional optical beam for nanooptical integrated circuits," Trans. Electron., vol. E90C, no. 1, pp. 87-94, Jan. 2007.

[2] R. J. Luebbers and F. Hunsberger, "FDTD for $N$ th-order dispersive media," IEEE Trans. Antennas Propag., vol. 40, no. 11, pp. 1297-1301, Nov. 1992.

[3] D. F. Kelley and R. J. Luebbers, "Piecewise linear recursive convolution for dispersive media using FDTD," IEEE Trans. Antennas Propag., vol. 44, no. 6, pp. 792-797, Jun. 1996.

[4] R. Siushansian and J. LoVetri, "A comparison of numerical techniques for modeling electromagnetic dispersive media," IEEE Microw. Guided Wave Lett., vol. 5, no. 12, pp. 426-428, Dec. 1995.

[5] R. Siushansian and J. LoVetri, "Efficient evaluation of convolution integrals arising in FDTD formulations of electromagnetic dispersive media," J. Electromag. Waves Applicat., vol. 11, no. 1, pp. 101-117, 1997.

[6] A. Vial, A.-S. Grimault, D. Macías, D. Barchiesi, and M. L. de la Chapelle, "Improved analytical fit of gold dispersion: Application to the modeling of extinction spectra with a finite-difference time-domain method," Phys. Rev. B, vol. 71, pp. 085416-1-7, Feb. 2005.

[7] E. Lidorikis, S. Egusa, and J. D. Joannopoulos, "Effective medium properties and photonic crystal superstructures of metallic nanoparticle arrays," J. Appl. Phys., vol. 101, no. 5, pp. 054304-1-7, Mar. 2007.

[8] W. H. P. Pernice, F. P. Payne, and D. F. G. Gallagher, "A general framework for the finite-difference time-domain simulation of real metals," IEEE Trans. Antennas Propag., vol. 55, no. 3, pp. 916-923, Mar. 2007.

[9] K.-Y. Jung and F. L. Teixeira, "Multispecies ADI-FDTD algorithm for nanoscale three-dimensional photonic metallic structures," IEEE Photon. Technol. Lett., vol. 19, no. 8, pp. 586-588, Apr. 15, 2007.

[10] T. Yamaguchi and T. Hinata, "Optical near-field analysis of spherical metals: Application of the FDTD method combined with the ADE method," Opt. Express, vol. 15, no. 18, pp. 11481-11491, Sep. 2007.

[11] F. Hao and P. Nordlander, "Efficient dielectric function for FDTD simulation of the optical properties of silver and gold nanoparticles," Chem. Phys. Lett., vol. 446, no. 1-3, pp. 115-118, Sep. 2007.

[12] J. Shibayama, R. Takahashi, J. Yamauchi, and H. Nakano, "Frequency-dependent locally one-dimensional FDTD implementation with a combined dispersion model for the analysis of surface plasmon waveguides," IEEE Photon. Technol. Lett., vol. 20, no. 10, pp. 824-826, May 15, 2008.

[13] J. Shibayama, M. Muraki, J. Yamauchi, and H. Nakano, "Efficient implicit FDTD algorithm based on locally one-dimensional scheme," Electron. Lett., vol. 41, no. 19, pp. 1046-1047, Sep. 2005.

[14] Y. Zhao, P. Belov, and Y. Hao, "Accurate modeling of the optical properties of left-handed media using a finite-difference time-domain method," Phys. Rev. E, vol. 75, no. 3, pp. 037602-1-4, Mar. 2007. 